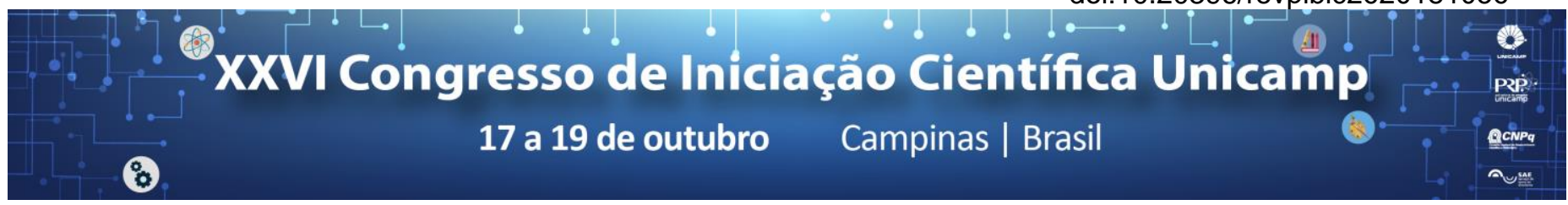

\title{
USING DEEP LEARNING TO PREDICT SOLAR EXPLOSIONS USING MAGNETOGRAMS
}

\section{Matheus E. R. Fernandes*, André Leon S. Gradvohl.}

\begin{abstract}
Solar activities, especially solar explosions, have a significant impact on some important technologies used on Earth, e.g., energy transmission networks and communications. Depending on the class of the explosion, the consequences can be hazardous. Therefore, when forecasting of solar explosions, earlier we can take actions to mitigate their impact on the affected technologies on Earth. In this work, we applied Deep Learning techniques to classify solar magnetograms, which indicate the class of a solar explosion. The classification of such images may help to anticipate the phenomenon. The results show a $97 \%$ of accuracy for the magnetograms classification.
\end{abstract}

\section{Key words:}

Solar explosions, Deep Learning, Classification.

\section{Introduction}

The Sun produces solar explosions by the breakdown of magnetic fields. These explosions have a significant impact on some of the technologies used on Earth. One of the instruments that allow anticipating these explosions are the magnetograms, i.e., images that show the variation of magnetic fields in the solar photosphere. In this project, we proposed the use of the Deep Learning technique to classify solar magnetograms, aiming at identifying, with the highest possible, accuracy when solar explosions will occur.

To classify the magnetograms automatically, we created a magnetogram database (GRADVOHL, FERNANDES 2017), collecting data from the Space Weather Live site (2017). Then, we balanced the dataset to make it possible to train the network with a proportional number of classes of explosions.

After that, we preprocessed the data to adapt it for training and testing the Deep Learning Neural Network models. We used the NVidia DIGITS software (2016) to train the neural networks, with the maximum performance through graphics processing units.

\section{Results and Discussion}

NVidia DIGITS software provided two tools to build and train the neural networks. One of them is Caffe, a Deep Learning framework created at UC Berkeley. Caffe was the first framework used because it is the default framework provided by the DIGITS software. The accuracy provided by Caffe trained models were good, most of them above the $70 \%$, the minimum established as a parameter. However, the loss of data within the training was as high as the accuracy, resulting in mistakes at classification and low accuracy in results.

DIGITS also provided another tool called Tensorflow. The use of the Tensorflow tool was a turning point for this research. We got better results as we progressed through the models. With Tensorflow we obtained a model named "Final", which is the best possible model we could reach. Table 1 depicts the results for training and testing 39 magnetograms as follows.
Table 1. Final model statistics.

\begin{tabular}{|c|c|c|c|c|c|}
\hline $\mathrm{P}$ & $\mathrm{N}$ & $\mathrm{TP}$ & $\mathrm{TN}$ & TPR & TNR \\
\hline 26 & 13 & 26 & 12 & 1 & 0.92 \\
\hline
\end{tabular}

Table 1 shows the following columns: $P$ (positive) is the number of images with explosions; $N$ (negative) is the number of images without explosion; TP is a true positive rate, indicating the number of positive images correctly classified; TN stands for true negative, representing the number of negative images correctly classified by the model. The last two columns are the true positive rate (TPR) and true negative rate (TNR).

After training and testing the Final model, we submitted 13 never seen magnetograms to the neural network to validate the model. The model was able to classify the whole group of true positive magnetograms correctly and missed only one true negative magnetogram.

\section{Conclusions}

The Final model reached the primary goal of this research, i.e., the correct classification of explosions with high accuracy and lowest error rate possible, with an overall of $97 \%$ accuracy and around $1 \%$ loss of data during training.

We can use the Final model to classify magnetograms in advance, which helps to forecast or to mitigate solar explosions impacts on Earth.

\section{Acknowledgement}

We would like to acknowledge the CNPq for the financial support of this project.

SPACE WEATHER LIVE. Top 50 solar flares. SpaceWeatherLive.com, 2017. Available at: https://www.spaceweatherlive.com/en/solar-activity/top-50-solarflares. Acess on january 3, 2018.

GRADVOHL, A. L. S \& FERNANDES, M. E. R. (2017). Samples of solar flares classes, active regions and time of occurrence (Version 0.1) [Dataset]. Available at: http://doi.org/10.5281/zenodo.1048995. Acess on january 7, 2018.

NVIDIA DIGITS. Interactive Deep Learning GPU Training System. NVIDIA Corporation, [2016]. Available at: https://developer.nvidia.com/digits. Acess on march 17, 2017. 